\title{
Transposition
}

Musique et Sciences Sociales

Hors-série 2 | 2020

Sound, Music and Violence

\section{Introduction. Son, musique et violence}

Introduction. Sound, Music and Violence

Luis Velasco-Pufleau

\section{(e) OpenEdition}

Journals

Édition électronique

URL : http://journals.openedition.org/transposition/5067

DOI : 10.4000/transposition.5067

ISSN : 2110-6134

\section{Éditeur}

CRAL - Centre de recherche sur les arts et le langage

\section{Référence électronique}

Luis Velasco-Pufleau, «Introduction. Son, musique et violence », Transposition [En ligne], Hors-série 2 2020, mis en ligne le 15 mars 2020, consulté le 15 avril 2020. URL : http://journals.openedition.org/ transposition/5067 ; DOI : https://doi.org/10.4000/transposition.5067

Ce document a été généré automatiquement le 15 avril 2020

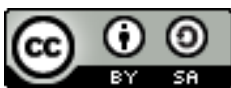

La revue Transposition est mise à disposition selon les termes de la Licence Creative Commons Attribution - Partage dans les Mêmes Conditions 4.0 International. 


\title{
Introduction. Son, musique et violence
}

\author{
Introduction. Sound, Music and Violence
}

Luis Velasco-Pufleau

1 Lorsqu'au troisième chant de la première partie de la Divine Comédie, Virgile fait franchir à Dante la porte de l'Enfer, ce dernier est effrayé non pas par ce qu'il voit, mais par ce qu'il entend. "Pleurs, soupirs et hautes plaintes résonnaient dans l'air sans étoiles $»^{1}$. L'horreur est telle que Dante commence à pleurer. Les lamentations des suppliciés dans « diverses langues » et « horribles jargons », les "mots de douleur » et "accents de rage», font un fracas tournoyant et assourdissant. Face à l'incompréhension de ces sons d'effroi et de douleur, Dante demande à Virgile : " Maître, qu'est-ce que j'entends ? $»^{2}$ Il ressent la douleur portée par les sons mais il ne sait pas qui les produit, ni pour quelle raison : il n'avait jamais entendu de pareils sons. Pour pouvoir attacher un sens global à ce nouveau monde sonore, il doit désormais écouter attentivement; car l'écoute sera essentielle pour explorer l'espace, pour comprendre les situations et pour donner sens à sa progression dans les différents cercles de l'enfer. Ceci jusqu'aux derniers vers, quand Dante et son guide trouvent la sortie du bas monde grâce à l'écoute : ils reconnaissent le chemin caché par où ils vont sortir " non par la vue mais par le son d'un petit ruisseau $»^{3}$ qui creuse la roche. L'empruntant, ils reviennent au monde clair et peuvent enfin « revoir les étoiles $»^{4}$.

2 Le récit que fait Dante de sa traversée de l'Enfer pointe la complexité émotionnelle des phénomènes sonores et montre comment l'écoute peut devenir un outil d'exploration, d'engagement et de connaissance sensible du monde. Ce numéro hors-série de Transposition propose d'explorer ces sujets à partir de l'analyse des liens entre son, musique et violence. Il entend ainsi contribuer, six ans après le numéro 4 consacré à "Musique et conflits armés après 1945 " $^{5}$ à l'essor considérable des recherches interdisciplinaires qui se donnent pour objectif la compréhension de la violence collective et de la guerre à partir du son et de la musique. En effet, dans les champs de la musicologie, de l'ethnomusicologie, de l'histoire, de l'anthropologie ou encore des sound studies, de nombreux travaux se sont intéressés aussi bien aux répertoires 
mobilisés en temps de guerre qu'aux expériences d'écoute de combattant.e.s et de civil.e.s en contexte de conflit ou post-conflit. Les travaux pionniers de Svanibor Pettan sur les guerres des Balkans ${ }^{6}$ ont été suivis par des travaux sur les liens entre musique et violence $^{7}$, des recherches sur les pratiques sonores et musicales des soldats durant ou après l'invasion des États-Unis en Irak ${ }^{8}$, de même que par un intérêt renouvelé pour les deux guerres mondiales ${ }^{9}$ et les conflits armés des $\mathrm{xIX}^{\mathrm{e}}$ et $\mathrm{Xx}^{\mathrm{e}}$ siècles ${ }^{10}$. Ces travaux constituent différentes entrées dans une " acoustémologie de la violence " ${ }^{11}$. Dans tous les cas, les sujets et les méthodes sont aussi divers que le nombre des chercheur.e.s impliqué.e.s.

3 Ce vaste projet scientifique est avant tout collectif et se place sous le signe du dialogue entre chercheur.e.s basé.e.s dans différents pays qui mobilisent des concepts et des méthodologies appartenant à plusieurs disciplines des sciences humaines et sociales. Ce numéro de Transposition paraît dans le contexte d'un mouvement social de grande ampleur, qui témoigne de nombreuses inquiétudes à l'égard des réformes néolibérales dans l'enseignement supérieur et la recherche en France. Pour parer au culte de la performance, au mythe de la réussite individuelle et aux injonctions à la compétition, il est important d'insister sur la dimension collective de la production des connaissances. Nous marchons sur des chemins qui ont été frayés par d'autres avant nous. Nos idées, aussi originales qu'elles puissent paraitre, font toujours partie de constellations plus vastes et sont redevables de l'héritage de personnes qui nous ont précédées et avec qui nous les avons développées. Comme le soulignait récemment le sociologue Gary Younge dans The Guardian, « seuls les privilégiés et les naïfs croient que les réalisations des gens sont purement le produit de leur propre génie $»^{12}$.

4 Ce numéro est collectif à plusieurs égards. Les trois articles du dossier thématique sont issus des journées d'étude Sound and Music in War from the Middle Ages to the Present, que j'ai eu l'opportunité d'organiser avec Marion Uhlig et Martin Rohde à l'Institut d'études médiévales de l'Université de Fribourg les 12 et 13 novembre $2018 .{ }^{13}$ La deuxième partie du numéro est composée d'un entretien et de trois commentaires critiques qui répondent et prolongent les propos énoncés. Enfin, la troisième partie est constituée de huit essais qui commentent certains textes du numéro ou développent des questions théoriques, éthiques et méthodologiques soulevées par la recherche sur la musique, le son et la guerre. Ces textes répondent à deux questionnements : de quelle façon l'étude du son et de la musique peut-elle aider à comprendre la violence collective et la guerre? Comment l'étude de la guerre et de la violence collective peut-elle aider à comprendre l'importance des pratiques musicales et de l'écoute pour les êtres humains ? $^{14}$

5 Toutefois, associer la musique à la violence, à la destruction et aux atrocités de la guerre ne va pas de soi dans la recherche en sciences humaines et sociales. Ainsi que le remarque l'ethnomusicologue Timothy Rice, les travaux dans le domaine de ce qu'il appelle " ethnomusicology in times (and places) of trouble » sont rares avant le début des années $2000^{15}$. Cela s'expliquerait selon lui, entre autres choses, par des imaginaires culturels dans lesquels la musique est forcément associée à des choses «bonnes » et par des présupposés scientifiques selon lesquels la musique ne peut être produite que dans des contextes socialement stables ${ }^{16}$. Les textes de ce numéro contribuent à la reconfiguration de ces croyances et, comme Morag J. Grant le suggère dans son essai, à l'exploration des fondements culturels de la guerre et de la violence collective. 


\section{Expériences d'écoute de la violence armée}

6 Le récit sonore de L'Enfer de Dante évoqué au début de cette introduction nous rappelle que le son peut constituer un événement qui modifie de façon durable la perception qu'un auditeur peut avoir du monde qui l'entoure. Les pleurs et les plaintes qui résonnent dans l'air sombre de l'Enfer terrifient Dante tout en lui faisant comprendre qu'il entre dans un lieu inconnu. Cependant, le son peut aussi être un processus qui se prolonge dans le temps et qui, par les interactions sensorielles avec la personne qui écoute, transforme sa perception du réel.

7 Dante apprend à écouter et à évoluer dans ce nouveau monde sonore et, par ce biais, développe de nouvelles connaissances sur son fonctionnement et les relations de pouvoir qui sont en jeu. La violence et la guerre déplacent les limites et les seuils des paysages sonores habituels, transformant durablement les repères et les capacités acoustiques des auditeurs熙trices ${ }^{17}$. Le développement de ces habitudes et aptitudes d'écoute constitue un nouveau «régime d'audition »: l'ensemble de techniques, de technologies, de régulations, de savoirs partagés qui donnent forme aux pratiques d'écoute d'une communauté donnée ${ }^{18}$.

8 Est-il possible d'essayer de comprendre l'expérience de la guerre à travers les régimes d'audition? Des recherches récentes ont exploré cette question, notamment à partir des récits de combattant.e. ${ }^{19}$. L'essai signé par Michael Guida dans ce numéro met en évidence une facette peu étudiée de ces récits : l'expérience sonore de la nature que font des soldats britanniques mobilisés sur le front de l'Ouest durant la Première Guerre mondiale. À travers l'analyse de sources diverses - journaux intimes, poèmes et lettres - Guida montre comment les soldats attachent une importance particulière aux chants d'oiseaux, qui encadrent leurs expériences d'écoute des tranchées ${ }^{20}$. Pour sa part, John Morgan O'Connell discute les idées développées par plusieurs auteur.e.s du numéro en les mettant en perspective avec ses propres recherches autour de la bataille de Gallipoli (1915-1916). Il explore les liens entre musique et mémoire, notamment lorsque la musique est utilisée pour remémorer et oublier, pour célébrer la victoire ou commémorer la défaite ${ }^{21}$.

9 L'analyse d'expériences d'écoute et de régimes d'audition a aussi permis d'étudier l'expérience de la violence armée de non-combattant.e. s2 $^{22}$.Ce domaine de recherche est exploré par Nikita Hock dans son article sur les expériences d'écoute de Juifs de Varsovie et de Galicie Orientale dans des abris souterrains durant la Seconde Guerre mondiale. Hock réussit à étudier, à partir d'un vaste corpus de journaux intimes, les expériences des civils - notamment des femmes et des personnes âgées - qui ont enduré la violence et la persécution durant l'Holocauste ${ }^{23}$.

\section{À l'écoute des vestiges sonores de la violence}

10 La question de l'accès aux sources et de leur analyse est centrale pour une acoustémologie de la violence. Comment interpréter les traces sonores de la guerre dans les sources écrites ? Ainsi que le souligne Annegret Fauser dans son essai, ces archives constituent des médiations d'expériences sonores du passé, des façons d'écouter et de mettre en récit. Prolongeant les travaux d'Ana María Ochoa Gautier, elle propose de suivre «une exploration accordée à l'acoustique [acoustically tuned] des 
archives écrites $»^{24}$ afin d'explorer les vestiges sonores de la violence, tout en questionnant les archives en tant qu'entités historiquement construites et, de ce fait, privilégiant les voix de certains types de témoins ${ }^{25}$.

Parce que la violence « est toujours une attaque à l'endroit de la dignité, du sens de soi, et du futur d'une personne $\aleph^{26}$, elle bouleverse et opère une reconfiguration des frontières entre son, bruit et silence, entre ce qui est dicible et ce qui ne l'est pas. Ainsi que l'affirme Ana María Ochoa Gautier, « une des caractéristiques de la violence est la redéfinition de l'espace acoustique $»^{27}$. Anna Papaeti explore les modalités et les enjeux pour la recherche en (ethno)musicologie de cette redéfinition dans le contexte de l'utilisation de la musique à des fins de torture. Elle réfléchit dans son essai aux conséquences du traumatisme infligé par le son et la musique dans des contextes de détention et à la dimension éthique inhérente à l'écoute du témoignage des victimes. ${ }^{28}$ Les modalités de ce que la voix peut exprimer, et les frontières entre son, bruit et silence, sont aussi quelques-unes des questions examinées par Sarah Kay dans son article sur les sirventes composés par Bertran de Born, l'un des plus célèbres troubadours de la seconde moitié du XII ${ }^{\mathrm{e}}$ siècle - qui figure d'ailleurs dans L'Enfer de Dante. Mobilisant le concept lacanien d'« extimité ", Kay s'intéresse à la dimension sonore de ces chansons politiques d'amour et de guerre qui révèlent une médiation entre bruit et musique, et reconfigurent la transmission poétique des sujets de l'amour et de la mort ${ }^{29}$.

Pour sa part, Martin Daughtry appelle à rompre avec une vision anthropocentrée de l'activité musicale ; il en questionne les cadres pratiques et théoriques qui contribuent à alimenter l'un des moteurs de la violence moderne : la dichotomie entre nature et culture $^{30}$. Avoir séparé des êtres qui devaient demeurer ensemble, voilà justement le reproche que Dante fait au troubadour Bertran de Born, lorsque son spectre apparaît au huitième cercle de l'enfer tenant « sa tête coupée par les cheveux, suspendue à la main comme une lanterne $»^{31}$ : divisé lui-même pour avoir semé la discorde, sa peine est d'avancer avec son cerveau séparé de son corps. La réflexion sur les liens entre musique et violence donne à Daughtry l'opportunité de penser un dépassement de l'exceptionnalisme humain et d'appeler à une autre écoute des vestiges sonores de la violence humaine.

13 L'écoute de ces vestiges peut soulever des questionnements éthiques importants quand les chercheur.e.s sont amené.e.s à s'entretenir et à travailler avec des personnes qui ont participé activement aux atrocités de la guerre. Quoique fondamentale, cette question reste relativement peu abordée de façon explicite dans les recherches sur les liens entre son, musique et violence. Hettie Malcomson développe cet enjeu, affirmant la nécessité de respecter l'humanité et la subjectivité des personnes impliquées, et d'éviter tout sensationnalisme dans le processus de production des connaissances ${ }^{32}$.

\section{Violence et agentivité du son et de la musique}

L'ensemble des textes de ce numéro partagent une position scientifique qu'il est utile de rappeler : le son et la musique ne sont pas étudiés comme la cause de l'action violente, mais plutôt comme des ressources symboliques que les acteurs peuvent mobiliser dans des processus ou des dynamiques de violence. La différence est de taille et sous-entend le refus d'une ontologie du son et de la musique dans laquelle la volonté humaine serait subordonnée à leurs supposés pouvoirs. Il s'agit plutôt de comprendre 
de quelle façon les personnes se saisissent de la musique et des phénomènes sonores pour donner sens à leur réalité dans des contextes de guerre ou pour justifier des actes de destruction et de violence.

La musique peut être un moyen de projeter, d'encadrer et de préparer l'affrontement avec l'ennemi. L'imaginaire qu'elle véhicule tout comme ses caractéristiques sonores peuvent être mobilisés par les acteur.e.s afin de s'engager dans une confrontation réelle ou imaginaire. Cette hypothèse est explorée par Victor A. Stoichita dans son article sur les expériences d'écoute des soldats étatsuniens et du terroriste norvégien Anders Breivik. Il montre de quelle façon la possibilité que le son et la musique se

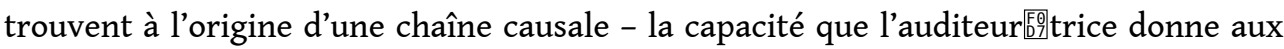
sons de "transformer " le monde dans lequel il ou elle habite - est étroitement liée à celle de l'ontologie de l'expérience d'écoute ${ }^{33}$. Cornelia Nuxoll explore d'autres enjeux de l'agentivité de la musique dans son essai sur son travail de terrain en Sierra Leone avec d'anciens combattants du Revolutionary United Front (RUF). Ses observations pointent la complexité émotionnelle liée à la musique utilisée dans des dynamiques de violence tout comme dans des processus de désarmement ${ }^{34}$.

L'entretien que j'ai réalisé avec Jean-Marc Rouillan, membre fondateur du groupe révolutionnaire armé Action directe (1977-1987), présente le point de vue d'un protagoniste d'une certaine violence politique. Il y raconte comment son engagement politique autour de 1968 a été précédé d'un engagement musical, dans l'attente d'un affrontement plus direct avec l'État. L'écoute de la musique rock et punk a servi de catalyseur pour fédérer des revendications de liberté et d'action politique autonome ${ }^{35}$. L'entretien est suivi de trois commentaires critiques de Matthew Worley (University of Reading) ${ }^{36}$, Timothy Scott Brown (Northeastern University) ${ }^{37}$ et Jeremy Varon (New School) $)^{38}$. Ces textes approfondissent, critiquent ou contextualisent des prises de position ou des faits exposés par Jean-Marc Rouillan. Qu'il s'agisse du rapport entre musique et mémoire de luttes politiques, de l'autonomie recherchée par le mouvement punk ou encore du lien entre rock et marchandisation, les commentaires apportent de précieux contrepoints pour saisir la complexité de la situation historique évoquée.

Comme le remarque Morag J. Grant, «bien après le cessez-le-feu, la musique continue de jouer un rôle souvent fondamental dans la célébration ou la commémoration de guerres et de guerriers, servant ainsi de boîte à outils pour la mémoire collective qui elle-même, bien trop souvent, est mobilisée au service des guerres à venir $»^{39}$. Ainsi, en s'intéressant aux liens entre son, musique et violence, ce numéro de Transposition interroge les façons dont les sociétés humaines se pensent, construisent leur mémoire collective et se projettent dans le futur.

\section{BIBLIOGRAPHIE}

ALIGHIERI Dante, La Divine Comédie : l'Enfer, RISSET Jacqueline (trad.), Paris, GF Flammarion, 2004. 
AUDOIN-ROUZEAU Stéphane, BUCH Esteban, CHIMÈNES Myriam et DUROSOIR Georgie (dir.), La Grande Guerre des musiciens, Lyon, Symétrie, 2009.

BIRDSALl Carolyn, Nazi Soundscapes: Sound, Technology and Urban Space in Germany, 1933-1945, Amsterdam, Amsterdam University Press, 2012.

BROWN Timothy Scott, « Going Underground: The Politics of Free Music around 1968 », Transposition, $\mathrm{n}^{\circ}$ Hors-série 2, 2020, https://doi.org/10.4000/transposition.4863.

CUSICK Suzanne G., " "You are in a place that is out of the world. ..": Music in the Detention Camps of the "Global War on Terror" ", Journal of the Society for American Music, vol. 2, n 1, 2008, p. 1-26.

DAUGHTRY J. Martin, « Did Music Cause the End of the World? », Transposition, ${ }^{\circ}$ Hors-série 2, 2020, https://doi.org/10.4000/transposition.5192.

DAUGHTRY J. Martin, Listening to War: Sound, Music, Trauma, and Survival in Wartime Iraq, New York, Oxford University Press, 2015.

EVANS Brad et LENNARD Natasha, Violence: Humans in Dark Times, San Francisco, City Lights Books, 2018.

FAST Susan et PEGLEY Kip, Music, Politics, and Violence, Middletown, Wesleyan University Press, 2012. FAUSER Annegret, « Sound, Music, War, and Violence: Listening from the Archive », Transposition, $\mathrm{n}^{\circ}$ Hors-série 2, 2020, https://doi.org/10.4000/transposition.4310.

FAUSER Annegret, Sounds of War: Music in the United States during World War II, New York, Oxford University Press, 2013.

GILMAN Lisa, My Music, my War: The Listening Habits of U.S. Troops in Iraq and Afghanistan, Middletown, Wesleyan University Press, 2016.

GRANT M. J. et PAPAETI Anna (dir.), « Music and Torture | Music and Punishment », The World of Music, vol. 2, n 1, 2013, https://www.jstor.org/stable/i24316991.

GRANT Morag Josephine, « On Music and War », Transposition, ${ }^{\circ}$ Hors-série 2, 2020, https:// doi.org/10.4000/transposition.4469.

GRANT Morag Josephine, MÖLLEMANN Rebecca, MORLANDSTÖ Ingvill, MÜNZ Simone Christine et NUXOLL Cornelia, « Music and Conflict: Interdisciplinary Perspectives », Interdisciplinary Science Reviews, vol. 35, n 2, 2010, p. 183-198.

GUIDA Michael, « Nature's Sonic Order on the Western Front », Transposition, $\mathrm{n}^{\circ}$ Hors-série 2, 2020, https://doi.org/10.4000/transposition.4770.

HARTFORD Kassandra, « Listening to the Din of the First World War », Sound Studies, vol. 3, n 2, 2017, p. 98-114.

HOCK Nikita, « Making Home, Making Sense: Aural Experiences of Warsaw and East Galician Jews in Subterranean Shelters during the Holocaust ", Transposition, $\mathrm{n}^{\circ}$ Hors-série 2, 2020, https:// doi.org/10.4000/transposition.4205.

JARDIN Étienne (dir.), Music and War in Europe from the French Revolution to WWI, Turnhout, Brepols, 2016.

JOHNSON Bruce et CLOONAN Martin, Dark Side of the Tune: Popular Music and Violence, Farnham, Ashgate, 2009. 
KALTENECKER Martin, « Paysage endivisionné. Notes sur les frontières acoustiques de la guerre », Transposition, $\mathrm{n}^{\circ}$ 6, 2016, https://doi.org/10.4000/transposition.1615.

KALTENECKER Martin, « “What Scenes! - What Sounds!” Some Remarks on Soundscapes in War Times ", JARDIN Étienne (dir.), Music and War in Europe from the French Revolution to WWI, Turnhout, Brepols, 2016, p. 3-27.

KAY Sarah, « Songs of War: The Voice of Bertran de Born », Transposition, $\mathrm{n}^{\circ}$ Hors-série 2, 2020, https://doi.org/10.4000/transposition.3785.

MALCOMSON Hettie, « On Sensationalism, Violence and Academic Knowledge », Transposition, ${ }^{\circ}$ Hors-série 2, 2020, https://doi.org/10.4000/transposition.4931.

MOORE Rachel, Performing Propaganda: Musical Life and Culture in Paris during the First World War, Woodbridge, The Boydell Press, 2018.

NUXOLL Cornelia, «Culprit or Accomplice: Observations on the Role and Perception of Music in Violent Contexts in the Sierra Leone War ", Transposition, $\mathrm{n}^{\circ}$ Hors-série 2, 2020, https://doi.org/ 10.4000/transposition.4382.

OCHOA GAUTIER Ana María, «El silencio como armamento sonoro », DE GAMBOA Camila et URIBE María Victoria (dir.), Los silencios de la guerra, Bogotá, Editorial Universidad del Rosario, 2017, p. 117-157. OCHOA GAUTIER Ana María, Aurality: Listening and Knowledge in Nineteenth-Century Colombia, Durham, Duke University Press, 2014.

OCHOA GAUTIER Ana María, «A manera de introducción: la materialidad de lo musical y su relación con la violencia », Trans, $n^{\circ}$ 10, 2006, https://www.redalyc.org/articulo.oa?id=82201001.

o'ConNell John Morgan, « Sound Bites: Music as Violence », Transposition, $\mathrm{n}^{\circ}$ Hors-série 2, 2020, https://doi.org/10.4000/transposition.4524.

o'CONNELl John Morgan et CASTELO-BRANCo Salwa el-Shawan (dir.), Music and Conflict, Urbana, University of Illinois Press, 2010.

PAPAETI Anna, "On Music, Torture and Detention: Reflections on Issues of Research and Discipline”, Transposition, no. Hors-série 2, 2020, https://doi.org/10.4000/transposition.5289.

PETIT Élise, Musique et politique en Allemagne: du III Reich à l'aube de la guerre froide, Paris, Presses de l'Université Paris-Sorbonne, 2018.

PETTAN Svanibor, Music, Politics, and War: Views from Croatia, Zagreb, Institute of Ethnology and Folklore Research, 1998.

PIESLAK Jonathan, Sound Targets: American Soldiers and Music in the Iraq War, Bloomington, Indiana University Press, 2009.

PILZER Joshua D., Hearts of Pine: Songs in the Lives of Three Korean Survivors of the Japanese « Comfort Women ", New York, Oxford University Press, 2012.

RICE Timothy, « Ethnomusicology in Times of Trouble », Yearbook for Traditional Music, vol. 46, 2014, p. 191-209.

SтогснітA Victor A., « Affordance to Kill: Sound Agency and Auditory Experiences of a Norwegian Terrorist and American Soldiers in Iraq and Afghanistan ", Transposition, $\mathrm{n}^{\circ}$ Hors-série 2, 2020 , https://doi.org/10.4000/transposition.4065.

sтогснітA Victor A., « Musicopathies. La musique est-elle bonne pour la santé ? », Terrain, $\mathrm{n}^{\circ} 68$, 2017, p. 4-25. 
SYKES Jim, « Ontologies of Acoustic Endurance: Rethinking Wartime Sound and Listening », Sound Studies, vol. 4, $\mathrm{n}^{\circ} 1,2018$, p. 35-60.

VARON Jeremy, « Reflections on a Revolutionary and Music », Transposition, $\mathrm{n}^{\circ}$ Hors-série 2, 2020, https://doi.org/10.4000/transposition.4644.

VELASCO PUFLEAU Luis, « De la musique à la lutte armée, de 1968 à Action directe : entretien avec Jean-Marc Rouillan », Transposition, $n^{\circ}$ Hors-série 2, 2020, https://doi.org/10.4000/transposition. 3709.

VELASCO-PUFLEAU Luis, « Quand il ne reste que la guerre pour tuer le silence : Écouter No One Is Innocent après le 13 novembre 2015 », Volume!, vol. 15, nº 2, 2019, p. 91-99.

VELASCO-PUFLEAU Luis, « No sound is innocent : réflexions sur l'appropriation et la transformation de l'expérience sonore de la violence extrême ", Filigrane, nº 23, 2018, https:// revues.mshparisnord.fr/filigrane/index.php?id=888.

VELASCO-PUfLEAU Luis (dir.), « Musique et conflits armés après 1945 », Transposition, nº 4, 2014, https://doi.org/10.4000/transposition.407.

VOLCLER Juliette, Le son comme arme : les usages policiers et militaires du son, Paris, La Découverte, 2011.

WILLIAMS Gavin (dir.), Hearing the Crimean War: Wartime Sound and the Unmaking of Sense, Oxford, Oxford University Press, 2019.

WORLEY Matthew, « Guitars Give Way to Guns: A Commentary on an Interview with Jean-Marc Rouillan », Transposition, n Hors-série 2, 2020, https://doi.org/10.4000/transposition.4284.

YOUNGE Gary, «In these Bleak Times, Imagine a World where You Can Thrive », The Guardian, https://www.theguardian.com/commentisfree/2020/jan/10/bleak-times-thrive-last-columnguardian, 10 January 2020, consulté le 25 février 2020.

\section{NOTES}

1. AlighiERI Dante, La Divine Comédie : l'Enfer, RISSET Jacqueline (trad.), Paris, GF Flammarion, 2004, p. 41.

2. Ibid., p. 43.

3. Ibid., p. 311.

4. Ibid.

5. velasco-Pufleau Luis (dir.), « Musique et conflits armés après 1945 », Transposition, $\mathrm{n}^{\circ} 4$, 2014, https://doi.org/10.4000/transposition.407.

6. PETTAN Svanibor, Music, Politics, and War: Views from Croatia, Zagreb, Institute of Ethnology and Folklore Research, 1998.

7. FAST Susan et PEGLEY Kip, Music, Politics, and Violence, Middletown, Wesleyan University Press, 2012 ; JoHnson Bruce et CLOONAN Martin, Dark Side of the Tune: Popular Music and Violence, Farnham, Ashgate, 2009 ; GRANT Morag Josephine, MÖLLEMANN Rebecca, MORLANDSTÖ Ingvill, MÜNZ Simone Christine et NUxoll Cornelia, «Music and Conflict: Interdisciplinary Perspectives ", Interdisciplinary Science Reviews, vol. 35, $\mathrm{n}^{\circ}$ 2, 2010, p. 183-198; o'CONNELL John Morgan et CASTELOBRANCO Salwa el-Shawan (dir.), Music and Conflict, Urbana, University of Illinois Press, 2010 ; осHOA GAUTIER Ana María, «A manera de introducción: la materialidad de lo musical y su relación con la violencia», Trans, $\mathrm{n}^{\circ}$ 10, 2006, https://www.redalyc.org/articulo.oa?id=82201001; STOICHITA Victor A., «Musicopathies. La musique est-elle bonne pour la santé ?», Terrain, $n^{\circ} 68,2017$, 
p. 4-25; VOLCLER Juliette, Le son comme arme: les usages policiers et militaires du son, Paris, La Découverte, 2011.

8. Voir par exemple, cusick Suzanne G., " "You are in a place that is out of the world. ..": Music in the Detention Camps of the "Global War on Terror" ", Journal of the Society for American Music, vol. 2, n' 1, 2008, p. 1-26 ; DAUGHTRY J. Martin, Listening to War: Sound, Music, Trauma, and Survival in Wartime Iraq, New York, Oxford University Press, 2015; GILMAN Lisa, My Music, my War: The Listening Habits of U.S. Troops in Iraq and Afghanistan, Middletown, Wesleyan University Press, 2016 ; PIESLAK Jonathan, Sound Targets: American Soldiers and Music in the Iraq War, Bloomington, Indiana University Press, 2009.

9. Voir par exemple, AUdoIN-ROUZEAU Stéphane, BUCH Esteban, CHIMÈNES Myriam et DUROSOIR Georgie (dir.), La Grande Guerre des musiciens, Lyon, Symétrie, 2009; BIRDSALl Carolyn, Nazi Soundscapes: Sound, Technology and Urban Space in Germany, 1933-1945, Amsterdam, Amsterdam University Press, 2012 ; FAUSER Annegret, Sounds of War: Music in the United States during World War II, New York, Oxford University Press, 2013 ; HARTFORD Kassandra, «Listening to the Din of the First World War », Sound Studies, vol. 3, n 2, 2017, p. 98-114; KALTENECKER Martin, "Paysage endivisionné. Notes sur les frontières acoustiques de la guerre ", Transposition, n 6, 2016, https:// doi.org/10.4000/transposition.1615; MOORE Rachel, Performing Propaganda: Musical Life and Culture in Paris during the First World War, Woodbridge, The Boydell Press, 2018 ; PETIT Élise, Musique et politique en Allemagne: du IIIe Reich à l'aube de la guerre froide, Paris, Presses de l'Université ParisSorbonne, 2018.

10. Voir par exemple, grant M. J. et PAPAETI Anna (dir.), «Music and Torture I Music and Punishment ", The World of Music, vol. 2, n 1, 2013, https://www.jstor.org/stable/i24316991; JARDIN Étienne (dir.), Music and War in Europe from the French Revolution to WWI, Turnhout, Brepols, 2016 ; OCHOA GAUTIER Ana María, « El silencio como armamento sonoro », DE GAMBOA Camila et URIBE María Victoria (dir.), Los silencios de la guerra, Bogotá, Editorial Universidad del Rosario, 2017, p. 117-157; SYKES Jim, «Ontologies of Acoustic Endurance: Rethinking Wartime Sound and Listening ", Sound Studies, vol. 4, $\mathrm{n}^{\circ} 1$, 2018, p. 35-60 ; williams Gavin (dir.), Hearing the Crimean War: Wartime Sound and the Unmaking of Sense, Oxford, Oxford University Press, 2019.

11. OCHOA GAUTIER, «A manera de introducción ».

12. "Only the privileged and the naive believe people's achievements are purely the product of their own genius ", YounGE Gary, "In these Bleak Times, Imagine a World where You Can Thrive ", The Guardian, https://www.theguardian.com/commentisfree/2020/jan/10/bleak-timesthrive-last-column-guardian, 10 January 2020, consulté le 25 février 2020.

13. Le programme complet est consultable ici https://agenda.unifr.ch/e/fr/4349

14. Je voudrais remercier les auteur.e.s qui ont participé à ce numéro spécial pour leur générosité et leur patience. Merci aussi aux relecteurs et relectrices anonymes qui ont contribué à travers leurs commentaires et leurs suggestions à améliorer les textes de ce numéro. Enfin, je voudrais exprimer ma gratitude aux membres du comité de rédaction de Transposition pour leur soutien dans ce projet ainsi qu'aux collègues et ami.e.s qui ont accueilli mes recherches à l'Université de Fribourg, à la Fondation Maison des sciences de l'homme (FMSH) et à l'Université de Berne.

15. RICE Timothy, «Ethnomusicology in Times of Trouble », Yearbook for Traditional Music, vol. 46, 2014, p. 191-209.

16. Ibid., p. 192.

17. KALTENECKER Martin, «"What Scenes! - What Sounds!” Some Remarks on Soundscapes in War Times ", JARDIN Étienne (dir.), Music and War in Europe from the French Revolution to WWI, Turnhout, Brepols, 2016, p. 3-27.

18. DAUGHTRY, Listening to War, p. 124.

19. DAUGHTRY, Listening to War ; GILMAN, My music, my War ; PIESLAK, Sound Targets. 
20. GUIDA Michael, « Nature's Sonic Order on the Western Front », Transposition, $\mathrm{n}^{\circ}$ Hors-série 2, 2020, https://doi.org/10.4000/transposition.4770.

21. o'ConNell John Morgan, « Sound Bites: Music as Violence ", Transposition, ${ }^{\circ}$ Hors-série 2, 2020, https://doi.org/10.4000/transposition.4524.

22. SYKES, « Ontologies of acoustic endurance »; PILZER Joshua D., Hearts of Pine: Songs in the Lives of Three Korean Survivors of the Japanese "Comfort Women ", New York, Oxford University Press, 2012 ; VELASCO-PUFLEAU Luis, « No sound is innocent : réflexions sur l'appropriation et la transformation de l'expérience sonore de la violence extrême", Filigrane, $\mathrm{n}^{\circ} 23$, 2018, https:// revues.mshparisnord.fr/filigrane/index.php?id=888; VELASCO-PUFLEAU Luis, "Quand il ne reste que la guerre pour tuer le silence : Écouter No One Is Innocent après le 13 novembre 2015 ", Volume!, vol. 15, n 2, 2019, p. 91-99.

23. носк Nikita, «Making Home, Making Sense: Aural Experiences of Warsaw and East Galician Jews in Subterranean Shelters during the Holocaust », Transposition, $\mathrm{n}^{\circ}$ Hors-série 2, 2020, https://doi.org/10.4000/transposition.4205.

24. "An acoustically tuned exploration of the written archive", осHOA GAUTIER Ana María, Aurality: Listening and Knowledge in Nineteenth-Century Colombia, Durham, Duke University Press, 2014 , p. 3.

25. FAUSER Annegret, "Sound, Music, War, and Violence: Listening from the Archive", Transposition, $\mathrm{n}^{\circ}$ Hors-série 2, 2020, https://doi.org/10.4000/transposition.4310.

26. "Is always an attack upon a person's dignity, sense of selfhood, and future », EVANs Brad et LENNARD Natasha, Violence: Humans in Dark Times, San Francisco, City Lights Books, 2018, p. 3.

27. «Una de las características de la violencia es la redefinición del espacio acústico », осноА GAUTIER, «A manera de introducción ».

28. PAPAETI Anna, "On Music, Torture and Detention: Reflections on Issues of Research and Discipline”, Transposition, no. Hors-série 2, 2020, https://doi.org/10.4000/transposition.5289.

29. KAY Sarah, « Songs of War: The Voice of Bertran de Born », Transposition, $\mathrm{n}^{\circ}$ Hors-série 2, 2020, https://doi.org/10.4000/transposition.3785.

30. DAUGHTRY J. Martin, « Did Music Cause the End of the World? », Transposition, ${ }^{\circ}$ Hors-série 2, 2020, https://doi.org/10.4000/transposition.5192.

31. ALIGHIERI, La Divine Comédie : l'Enfer, p. 259.

32. MALCOMSON Hettie, « On Sensationalism, Violence and Academic Knowledge », Transposition, $\mathrm{n}^{\circ}$ Hors-série 2, 2020, https://doi.org/10.4000/transposition.4931.

33. sтоіснітA Victor A., "Affordance to Kill: Sound Agency and Auditory Experiences of a Norwegian Terrorist and American Soldiers in Iraq and Afghanistan ", Transposition, $\mathrm{n}^{\circ}$ Hors-série 2, 2020, https://doi.org/10.4000/transposition.4065.

34. NUXoll Cornelia, «Culprit or Accomplice: Observations on the Role and Perception of Music in Violent Contexts in the Sierra Leone War ", Transposition, $n^{\circ}$ Hors-série 2, 2020, https://doi.org/ 10.4000 /transposition. 4382 .

35. Velasco PUfLEAU Luis, « De la musique à la lutte armée, de 1968 à Action directe : entretien avec Jean-Marc Rouillan», Transposition, $\mathrm{n}^{\circ}$ Hors-série 2, 2020, https://doi.org/10.4000/ transposition.3709.

36. WORLEY Matthew, « Guitars Give Way to Guns: A Commentary on an Interview with Jean-Marc Rouillan ", Transposition, ${ }^{\circ}$ Hors-série 2, 2020, https://doi.org/10.4000/transposition.4284.

37. BRown Timothy Scott, "Going Underground: The Politics of Free Music around 1968 ", Transposition, $\mathrm{n}^{\circ}$ Hors-série 2, 2020, https://doi.org/10.4000/transposition.4863.

38. VARON Jeremy, "Reflections on a Revolutionary and Music », Transposition, ${ }^{\circ}$ Hors-série 2, 2020, https://doi.org/10.4000/transposition.4644.

39. «Long after ceasefire, music continues to play an oftentimes fundamental role in celebrating or commemorating wars and warriors, thus functioning as a fundamental toolkit for collective 
memory which itself, all too often, becomes mobilised in the service of wars yet to come ", GRANT Morag Josephine, «On Music and War », Transposition, ${ }^{\circ}$ Hors-série 2, 2020, https://doi.org/ 10.4000/transposition.4469.

\section{RÉSUMÉS}

L'écoute peut devenir un outil d'exploration, d'engagement et de connaissance sensible du monde. La musique peut être un moyen de projeter, d'encadrer et de préparer l'affrontement avec l'ennemi. De quelle façon l'étude du son et de la musique peut-elle aider à comprendre la violence collective et la guerre ? Comment l'étude de la guerre et de la violence collective peutelle aider à comprendre l'importance des pratiques musicales et de l'écoute pour les êtres humains ? Ce numéro hors-série de Transposition propose d'explorer ces questions à partir de l'analyse des liens entre son, musique et violence.

Listening can become a tool for exploration of, engagement with and sensorial knowledge of the world. Music can be a device for projecting, framing and preparing for confrontation with the enemy. How can the study of sound and music help us to understand collective violence and war? How can the study of war and collective violence help us to understand the importance of musical practices and listening for human beings? This special issue of Transposition explores these questions through an analysis of the links between sound, music and violence.

\section{INDEX}

Keywords : agency, auditory regime, Dante, listening, music, sound, violence, war

Mots-clés : agentivité, Dante, écoute, guerre, musique, régime d'audition, son, violence

\section{AUTEUR}

\section{LUIS VELASCO-PUFLEAU}

Musicologue et artiste sonore, Luis Velasco-Pufleau est chercheur au Walter Benjamin Kolleg et à l'Institut de musicologie de l'Université de Berne. Son travail constitue une réflexion critique sur les liens entre musique et politique dans les sociétés contemporaines. En tant que chercheur et artiste sonore, il explore des formes d'écriture innovantes à la croisée de la création artistique et de la recherche en sciences humaines et sociales. ORCID : https://orcid.org/0000-0002-1330-974X 